\title{
Carnets de géographes
}

12 | 2019

Géographie(s) des mobilisations

Résister en situation autoritaire : le cas des collectifs militants d'après-Gezi à Istanbul (2013-2018)

\section{Agathe Fautras}

\section{CpenEdition}

Journals

Electronic version

URL: http://journals.openedition.org/cdg/4916

ISSN: 2107-7266

\section{Publisher}

UMR 245 - CESSMA

\section{Electronic reference}

Agathe Fautras, «Résister en situation autoritaire : le cas des collectifs militants d'après-Gezi à Istanbul (2013-2018) », Carnets de géographes [Online], 12 | 2019, Online since 20 December 2019, connection on 06 January 2020. URL : http://journals.openedition.org/cdg/4916

This text was automatically generated on 6 January 2020.

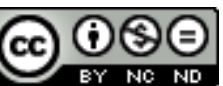

La revue Carnets de géographes est mise à disposition selon les termes de la Licence Creative Commons Attribution - Pas d'Utilisation Commerciale - Pas de Modification 4.0 International. 


\title{
Résister en situation autoritaire : le cas des collectifs militants d'après- Gezi à Istanbul (2013-2018)
}

\author{
Agathe Fautras
}

1 En Turquie, en mai-juin 2013, le mouvement de contestation du « parc de Gezi » réunit au plus fort de la mobilisation des centaines de milliers de personnes à Istanbul et se répand à travers toute la Turquie. Il commence par l'opposition d'un petit nombre de militante's écologistes et des luttes urbaines ${ }^{1}$ au déracinement d'arbres dans le parc de Gezi, prélude à la reconstruction de casernes ottomanes et à la réalisation du projet de piétonisation de la place Taksim attenante au parc. Puis converge vers la place et le parc une large foule, venue s'opposer à « l'attitude autoritaire du Premier ministre » (à l'époque Tayyip Erdoğan, devenu par la suite Président de la République de Turquie), à l'usage de la "force disproportionnée par la police envers les manifestants », et à la

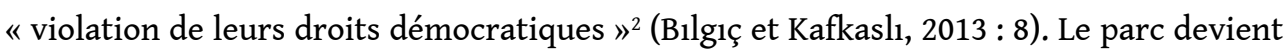
un lieu de rassemblement et de convergence de causes indépendantes les unes des autres - parmi lesquelles une meilleure considération des citoyen'ne's alévie·s (groupe confessionnel hétérodoxe dont certains éléments de culte sont d'origine chiite et préislamique), l'indemnisation des personnes subissant les projets de transformation urbaine, la reconnaissance de la communauté $\mathrm{LGBTQ}^{+}$, la fin du contrôle du corps des femmes et de la pression sur les artistes et les universitaires (Massicard, 2013) - qui témoignent d'une opposition à la politique du Parti de la Justice et du Développement (Adalet ve Kalkinma Partisi, AKP). L'occupation de la place Taksim et du parc de Gezi dure 11 jours, avant que les manifestanteess ne soient violemment évacuée's par la police. Au total, huit personnes sont mortes et des milliers ont été blessées lors des affrontements. Le parc de Gezi n'a certes pas été détruit, mais les revendications n'ont pas abouti.

2 Cette répression confirme le «tournant autoritaire » (Esen et Gümüşçü, 2016:5) amorcé dans ces années 2010, faisant suite à une période d'ouverture démocratique depuis l'arrivée au pouvoir en 2002 de l'AKP et de son chef de file Tayyip Erdoğan ${ }^{3}$ qui fait « figure de parenthèse dans l'histoire politique turque » (Doronsorro, $2017: 12$ ). En 
avril 2017, la réforme constitutionnelle votée par referendum entérine "une dérive vers un régime autoritaire total » (Çalışkan, $2018: 25)$, entamée de facto depuis la mise en place de l'état d'urgence suite à la tentative de coup d'État manquée du 15 juillet 2016. La nouvelle constitution fait entrer en vigueur un «nouveau système dans lequel les freins et les contrepoids fondamentaux et la séparation des pouvoirs (...) sont radicalement compromis » (ibid.:27). Si, lors du mouvement, la Turquie ne peut être qualifiée de régime démocratique, elle ne peut pas non plus être considérée comme un régime autoritaire stricto sensu: les élections nationales et législatives ont lieu régulièrement et sont globalement épargnées par la fraude, et aucun parti d'opposition majoritaire n'est ponctuellement interdit. Levitsky et Way (2002) ont analysé ces régimes hybrides, comme la Hongrie et la Russie, et formalisé le concept de "régime autoritaire compétitif » : malgré un système électoral globalement performant ${ }^{4}$, le parti au pouvoir abuse régulièrement des ressources étatiques ${ }^{5}$, empêche la couverture médiatique équitable de l'opposition et harcèle ses candidats ${ }^{6}$, et les porte-voix de l'opposition, tels que les militant·es, journalistes et universitaires sont régulièrement menacée's ou arrêtée's. Cette approche institutionnelle, particulièrement développée en science politique, permet de catégoriser la variété des régimes non-démocratiques apparus dans les années 1970-1990 (Brooker, 2009) et d'appréhender les mécanismes systémiques de l'autoritarisme. Néanmoins, des travaux récents insistent davantage sur l'importance de l'analyse des pratiques autoritaires. Ils s'intéressent à la fabrique quotidienne des rapports de pouvoir entre les différents échelons étatiques et l'opposition, qui s'effectuent sous différentes modalités (tolérance, conflictualité, négociation) et selon des temporalités spécifiques (nouveaux effets de contexte), impliquant ainsi des contraintes variées sur les acteurs (Planel, 2015 ; Glasius, 2018). Cette approche permet ainsi d'appréhender la production d'un " espace autoritaire " (Planel, 2015) qui passe par des pratiques quotidiennes de contrôle et de mise en ordre de l'espace physique. Afin de prendre en compte ces nuances, je parle donc plutôt de « situation autoritaire » (Piron, 2013 ; NA, 2016).

3 Un régime autoritaire cherche à produire un discours dominant qui légitime son existence et rende toute alternative politique impossible en « influençant et façonnant le vaste assemblage de textes oraux et écrits, de rituels, de culture visuelle et de performance qui constituent le discours public $»^{7}$ (Lewis, $2013: 333$ ). Toutefois, un tel régime ne se contente pas de discours et le matérialise dans l'espace physique, qui n'est pas un "pur "contenant" du politique ", mais un élément qui peut « limiter les champs du possible en politique autant qu'il peut les ouvrir» (Dikeç, 2009:1). Le discours public officiel sous le mandat de l'AKP présente une dimension idéologique, au sens d'un « ensemble de croyances concernant le bon ordre de la société et les moyens par lesquels celui-ci peut être atteint $»^{8}$ (Erikson et Tedin, 2003:64) : celui-ci peut être dit conservateur, islamique, nationaliste et néolibéral ${ }^{9}$. Or, selon Henri Lefebvre, «ce que l'on nomme "idéologie" n'acquiert de consistance qu'en intervenant dans l'espace social, dans sa production, pour y prendre corps " (Lefebvre, 1974:55). C'est notamment dans l'espace public qu'un discours dominant se matérialise, dans la mesure où celui-ci est à la fois métaphorique (porteur de discours, au sens habermassien de "sphère publique ») et urbanistique (catégorie d'action urbaine). En ce sens, si la vaste politique de réaménagement urbain d'Istanbul initiée dans les années 2000 s'explique par diverses intentions politiques, l'une d'elles est certainement la matérialisation du discours officiel dominant, visant à promouvoir une idéologie spécifique et à guider les pratiques sociales des usager-es. La reconfiguration urbaine 
de la place Taksim et du parc de Gezi en est justement une illustration. Si les précédents aménagements urbains de la place et du parc témoignaient d'une réécriture historique marquée par le récit républicain ${ }^{10}$, le projet de réaménagement de 2013 marque au contraire la superposition d'une nouvelle « couche de sens » (Pérouse, 2013a). Ainsi, la reconstruction des baraquements, leur transformation en espace commercial et l'édification d'une mosquée sont respectivement : des références à l'Empire ottoman, la promotion d'un certain consumérisme et une référence islamique.

4 Néanmoins, la fabrique de l'espace public officiel peut être contestée: lors de l'occupation de la place Taksim et du parc, les manifestantees ont créé un espace éphémère porteur de contre-discours, matérialisant des pratiques de solidarité, de démocratie directe et d'ouverture politique. Le mouvement a été réprimé et tenu en échec, et la situation autoritaire s'est progressivement durcie, rendant l'espace traditionnel de contestation (la rue, la place) de moins en moins accessible pour contester le discours public officiel et la manière dont il participe à façonner l'espace et les pratiques ${ }^{11}$. Dans une telle situation, comment l'opposition s'est-elle maintenue et sous quelles modalités spatiales? Verta Taylor pointe le fait que les chercheureuse's étudient davantage les "cycles de mobilisation de masse ", et s'intéressent peu "aux mouvements en état stationnaire ou en phase de déclin» (Taylor, 1989:249). Cela permet pourtant de comprendre les ruptures et continuités entre les cycles de mobilisation. L'autrice parle de "mise en veille» des mouvements sociaux, pour qualifier ce processus de maintien dans des environnements politiques hostiles, jouant un rôle de passeur entre deux mobilisations. À Istanbul, après la répression, les manifestant-e's se sont dispersée's dans différents quartiers de la ville et y ont tenu des forums tout l'été, dans des parcs ou des places. Entre 2013 et 2014, voyant leur fréquentation diminuer, les plus investiees ont créé des "collectifs", groupes de quelques dizaines de personnes fonctionnant de manière antihiérarchique et militant à différentes échelles (quartier, arrondissement, métropole) sur certaines questions représentées lors du mouvement du parc de Gezi : urbaines, alimentaires, écologiques, droits des femmes, de la communauté LGBTQ+ et des réfugiéee-s ${ }^{12}$. Ces collectifs sont pour la majeure partie informels, c'est-à-dire qu'ils ne se constituent pas en association officielle. Cette institutionnalisation présente en effet des désavantages: structure perçue comme hiérarchique par les militante's, lourdeur administrative, contrôle étatique. Si certains collectifs font néanmoins le choix de l'institutionnalisation, c'est afin de simplifier les démarches pour louer un local, afin d' "avoir un lieu à soi » qui puisse être utilisé de façon autonome, soit « de la façon que l'on veut, avec qui on veut, au moment où on veut» (Ripoll, 2005a:545). Ils cherchent ainsi à s'insérer dans l'espace urbain afin de faciliter l'organisation d'activités militantes et de mieux éviter la répression. Nancy Fraser parle d' " arènes discursives parallèles » qui permettent aux acteur.rice's de l'opposition ${ }^{13} \mathrm{~d}$ ' « élaborer et [de] diffuser des contre-discours, afin de formuler leur propre interprétation de leurs identités, leurs intérêts et leurs besoins " (Fraser, 1992 : 138). Elle ne soulève toutefois pas la question de leur ancrage spatial. L'objectif de cet article est double: contribuer à l'étude des mobilisations en analysant les formes de continuation d'un mouvement social en période de «mise en veille »; enrichir l'étude de la dimension spatiale de l'action collective, renouvelée en France dans le champ de la géographie dans les années 2000 (Ripoll et Veschambre (coord.), 2004), d'une part en mettant en lumière la dimension spatiale des stratégies élaborées par les militante's pour investir l'espace urbain dans une situation autoritaire, d'autre part en démontrant qu'ils participent à la production de l'espace 
urbain, dans des « contre-espaces publics» (Spurk, 2016) défiant les pratiques normées de l'espace public officiel dominant.

Si la recherche entreprise porte sur vingt collectifs, je m'appuierai dans cet article sur le travail de terrain réalisé auprès de deux collectifs créés à l'été 2013 et se consacrant aux causes écologistes et féministes : le collectif de la Protection des Forêts du Nord (Kuzey Ormanları Savunması), qui a pour objectif de protéger les forêts de la région d'Istanbul contre plusieurs projets et mégaprojets ${ }^{14}$; le Forum des femmes de Yoğurtçu (Yoğurtçu Kadı $n$ Forumu), qui réunit des femmes chaque semaine pour discuter de différents sujets et participe régulièrement à diverses mobilisations, notamment les marches du 8 mars (Journée internationale des droits des femmes) et du 25 novembre (Journée de lutte contre les violences faites aux femmes). Ces deux collectifs ont été spécifiquement choisis pour les raisons suivantes : ils ont été créés juste après le mouvement du parc de Gezi et sont encore actifs aujourd'hui, ils constituent la fourchette haute en terme de nombre de militante's impliquée's, et mènent et/ou participent à des actions qui ont une certaine efficacité politique.

6 Le travail de terrain s'appuie sur une recherche ethnographique: récits de vie et entretiens semi-directifs avec les militante-s, observation participante de réunions et d'événements qu'ils organisent, consultation de sources écrites (réseaux sociaux, archives, journaux). Les enquêtée's étaient informé·e's de ma qualité de chercheuse et du travail effectué (« recherche ouverte »), bien que la fluctuation de la fréquentation du collectif ne permette pas le consentement permanent de la totalité du groupe (Uldam et McCurdy, 2013). Ce travail de terrain a été initié en Master 2 dans le cadre d'un mémoire sur l'agriculture urbaine militante (entre octobre 2013 et mai 2015) et approfondi en thèse lors de trois séjours (de février à août 2016, de février à juin 2017, d'octobre 2017 à avril 2018). Il est évident que ces luttes ne sont pas nées en 2013 et témoignent d'une longue expérience militante. Néanmoins, le mouvement du parc de Gezi, par son ampleur et l'évolution des modes organisationnels, des répertoires d'action et des alliances politiques qu'il a provoquée, me semble présenter un intérêt particulier.

\section{Enjeux et contestations de l'espace public officiel à Istanbul}

\section{L'espace urbain stambouliote : entre production officielle et catalyseur de résistances}

7 Dans son article sur le mouvement du parc de Gezi, Benoît Montabone (2013) constate l'existence d'un ordre moral urbain qu'il décline sous le triptyque suivant: néolibéralisme économique, conservatisme religieux et néo-ottomanisme culturel. Ces idéologies sont intimement liées à la fabrique de la ville par l'État et se reflètent dans l'espace urbain par la promulgation de lois visant à guider les pratiques des usagerees et par la conception de projets urbains régis par des règles urbanistiques spécifiques. $\mathrm{Si}$ les premières politiques néolibérales en Turquie remontent aux années 1980, l'arrivée au pouvoir de l'AKP marque un nouveau tournant, particulièrement à travers la politique de transformation urbaine (kentsel dönüşüm), qui marie des projets de planification urbaine et l'expansion du secteur privé de la construction. Celle-ci a abouti à la marchandisation de l'espace urbain, devenue l'une des sources principales 
d'accumulation de capital via la rente foncière et la spéculation immobilière (Erdi Lelandais, 2017 :55). L'autoritarisme du gouvernement se manifeste par ailleurs dans la planification urbaine : alors qu'il ambitionne de faire d'Istanbul « une grande place internationale touristique, culturelle et financière » (Pérouse, 2013b), il lance des mégaprojets sans consulter la Grande Municipalité d'Istanbul (iBB) ni la population. Des populations précaires et parfois migrantes sont également expropriées des quartiers de gecekondu (habitations de fortune) où elles vivent, en utilisant notamment le prétexte du risque sismique (Morvan, 2013). Par le développement de multiples centres commerciaux, l'AKP tend aussi à produire un " conservateur qui consomme en famille " (Pérouse, 2013b). Concernant le conservatisme, l'AKP produit des espaces publics « régis par des normes de type islamique» (Erdi Lelandais, 2017:58). L'État guide également les pratiques urbaines par des législations et des discours conservateurs, portant par exemple sur le rôle et les pratiques des femmes dans l'espace public. Enfin, le discours politique officiel est émaillé de références nostalgiques à l'Empire ottoman, notamment pour justifier la démesure des projets urbains initiés. La politique urbaine vise donc à produire des espaces publics porteurs du projet politico-social et économique de l'AKP.

8 Cependant, Istanbul est également un catalyseur de résistances. En effet, la ville ainsi idéologiquement produite donne à voir la «déconnexion nette entre les attentes en termes d'égalité des droits et de traitement d'un groupe, et les expériences quotidiennes qui violent ces attentes » (Miller \& Nicholls, $2013: 458$ ). Par ailleurs, la ville est aussi un lieu de concentration des ressources politiques, économiques, sociales et culturelles, qui permet aux habitant·e's d'acquérir connaissances et compétences sur les mécanismes de dépossession et les stratégies de contestation. Les flux humains, qu'il s'agisse de la circulation de touristes, chercheur·ess, journalistes, étudiant·es évoluant loin du cercle familial, migrant·e's ou encore de militant·es étranger·e's, créent ainsi un "incubateur relationnel» (Nicholls, 2008) où les opportunités de rencontres et d'échanges sont propices à la circulation des idées et des expériences. La concentration d'une large population (15 millions d'habitante-s) permet aussi « un environnement favorable pour créer des alliances complexes entre diverses organisations, groupes et acteurs » (Miller \& Nicholls, $2013: 459)$. Enfin, la spécificité des villes turques réside dans l'existence d'une forte identité de quartier (mahalle), qui recouvre des valeurs d'appartenance, de familiarité et d'intimité spatiale (Mills, 2006). Après le mouvement du parc de Gezi, le mahalle a été particulièrement réinvesti « en tant qu'échelle et cadre de la contestation » par la gauche (Pérouse, 2014) ${ }^{15}$. À cette échelle, les militant·e·s ont poursuivi la production de contre-discours dans des forums et ont entamé la contestation de projets urbains locaux. Si le quartier est une base de politisation, il n'en est cependant pas le seul horizon (Castells, 1983) et les discours militants portent des revendications à diverses échelles. En somme, la fabrique actuelle d'Istanbul est perçue par les acteurrice's de l'opposition comme une dégradation de la vie urbaine (fermetures de l'espace, privatisation, contrôles spatiaux, espaces normatifs, surveillance, expropriations) (Harvey, 2012) et la vitalité de groupes militants, stimulés par un environnement urbain favorable, explique l'existence de fortes contestations.

\section{Qui sont les militant·es des collectifs ?}

9 Dans les années 2010, de grandes villes ont été le théâtre d'événements contestant le système néolibéral comme à Madrid, New-York, Athènes, ou critiquant les régimes en 
place, au Caire ou à Tunis. Pleyers et Glasius (2013) montrent que tous ces événements entrent en résonnance par l'articulation de trois valeurs au cœur des revendications : démocratie, dignité et justice sociale. Ceux-ci ont par ailleurs la particularité d'aboutir à l'appropriation éphémère d'espaces publics au sens urbanistique: places, rues et parcs (Spurk, 2016). En Turquie, ce moment exceptionnel a créé une «expérience commune fondamentale $\aleph^{16}$ pour une jeune génération n'ayant pas connu les années $1980^{17}$, au fondement d'une culture politique qui présente des nouveautés. Dès lors, s'il existe depuis longtemps des mouvements d'opposition en Turquie (şimşek, 2004), ceuxci s'étoffent en 2013 avec la création de collectifs aux valeurs politiques communes (anticapitalisme, droits humains, défense des minorités) mais aussi aux mêmes aspirations organisationnelles (diversité politique et sociale, horizontalité, mobilisation des TIC). Ces dernières avaient certes déjà émergé avec le mouvement altermondialiste (Erdi Lelandais, 2005) mais ont connu une effervescence dans la période post-Gezi.

Les entretiens réalisés auprès de 41 militante-s membres de l'ensemble des collectifs étudiés montrent qu'ils ont généralement entre 18 et 35 ans et situent en majorité leur éveil politique au moment du mouvement du parc de Gezi, bien que d'autres plus âgéees ont une longue expérience militante et sont souvent passéers par d'autres formes d'organisation (syndicats, partis politiques, associations) où ils ont certes acquis un capital militant mais s'en sont détournée's du fait, est-il dit, de leur structure trop rigide et hiérarchique, et de la faible place accordée aux initiatives individuelles. Par ailleurs, les militantees de tous âges sont surtout issuees de la nouvelle classe moyenne. Pour Keyder (2013a, 2013b), celle-ci s'est formée ces quinze dernières années avec

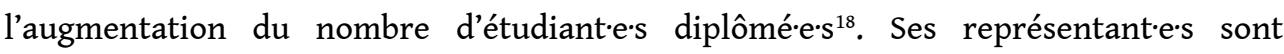
embauchée's "dans des lieux de travail relativement modernes, avec du temps de loisirs et des habitudes de consommation similaires à leurs pairs à l'échelle mondiale " ${ }^{19}$ (Keyder, 2013b). Les militante-s sont donc par exemple des étudiante·s à l'université, " cols blancs» ou salariée's des fonctions publiques (professeur'e's, universitaires), autant de positions supposant et offrant un solide capital culturel. Hommes et femmes sont assez également représentée's, bien que certains collectifs aient leur spécificité. Les coopératives alimentaires sont par exemple davantage tenues par des femmes, tandis qu'un autre collectif écologiste est principalement composé d'hommes. Toutefois, beaucoup de femmes se réunissent pour former une section féminine du collectif dont elles font partie afin de formuler leurs propres intérêts et besoins. Les origines géographiques sont quant à elle assez variées. Bien qu'une large part soit née à Istanbul, après que leurs parents y ont migré20, beaucoup viennent d'autres villes turques afin d'effectuer leurs études dans les bonnes universités, sans leur famille, ce qui leur permet une certaine liberté d'action et de reconfiguration de leurs identités. Beaucoup résident dans les arrondissements centraux d'Istanbul (Beyoğlu, Kadıköy, Sarıyer, Üsküdar, şişli), notamment dans des quartiers fortement ancrés à gauche, comme Okmeydanı. Bien que leurs identités politiques présentent une certaine diversité, difficilement envisageable avant le mouvement du parc de Gezi, elles restent surtout ancrées dans l'extrême-gauche et la mouvance anarchiste, et dans une moindre mesure dans une gauche à spectre large ${ }^{21}$. Ainsi, lors des entretiens, les enquêtée's se sont définie's comme "kémaliste", "écologiste ", " communiste ", "marxiste ", "socialiste", " anarchiste ", "de gauche ». Tou'te's ont en commun une critique du système capitaliste et des relations sociales et économiques qu'il produit, comme de toute forme d'impérialisme. À la question: "Quel parti s'approche le plus de vos valeurs politiques?», la majorité des enquêtée's répond le Parti Démocratique du 
Peuple (Halk Demokrasi Partisi, HDP), un parti pro-kurde rassemblant diverses mouvances de gauche, et dans une moindre mesure le Parti Républicain des Peuples (Cumhuriyet Halk Partisi, CHP), parti kémaliste. Enfin, les militante's anarchistes déclarent ne voter pour personne.

11 La majorité des collectifs créés après le mouvement de Gezi sont donc constitués de militantees de la nouvelle classe moyenne et habitent les quartiers centraux d'Istanbul. Si le mouvement du parc de Gezi ne s'est pas limité à l'occupation du parc et de la place, et si des actions ont aussi été menées dans des quartiers périphériques et populaires, la création de tels collectifs (antihiérarchique, revendiquant une filiation avec Gezi) n'y a pas été observée, de sorte que les militante-s ne représentent qu'une partie spécifique des manifestant-e's mobilisée's lors du mouvement de Gezi. Cette absence de collectifs s'explique sans doute d'une part par des effets de concurrence (Pérouse, 2017), dans la mesure où certains quartiers périphériques et ultra-périphériques sont déjà investis par des associations préexistantes œuvrant par exemple pour la lutte contre la transformation urbaine, ou par des groupes radicaux kurdes et d'extrême-gauche turque (dans les quartiers d'Okmeydanı et de Gazi); d'autre part par une distance sociale entre les habitante's de ces quartiers et ces représentant·e's de la nouvelle classe moyenne. Le mouvement du parc de Gezi a donc été approprié différemment selon les contextes sociaux et géographiques.

12 Ces groupes militants disposent donc de diverses ressources pour élaborer des critiques globales du modèle de société dans lequel ils vivent et pour organiser des contestations. Expérimentant ou assistant à la dégradation de la vie urbaine et à l'imposition de normes dans l'espace public officiel, ils développent différentes stratégies pour produire des discours d'opposition mais doivent adapter leurs actions à la situation autoritaire qui aboutit à la mise en place d'un contrôle plus strict de l'espace public.

\section{Porter une contestation dans l'espace public en situation autoritaire}

\section{Échelles et opportunités d'action militante dans l'espace public}

13 La période 2013-2018 est marquée par une accentuation des mesures autoritaires visant les acteur.rice's de l'opposition, qu'il s'agisse de partis politiques, d'associations officielles ou de collectifs informels ${ }^{22}$. Toutefois, les collectifs étudiés continuent de contester le discours officiel dans l'espace public. Ils doivent néanmoins déployer des stratégies pour contourner les interdictions notamment entérinées par la promulgation de l'état d'urgence. L'une des plus employées est sans doute la mobilisation du droit constitutionnel à tenir une "conférence de presse » dans l'espace public. Ayşen Uysal (2005) a déjà mis en évidence cette stratégie qui consiste à faire une déclaration publique sur un sujet spécifique. Elle permet tout autant de rassembler un petit nombre de militante's qu'un nombre indéfini de participante's sans avoir à déclarer une manifestation en préfecture. Une militante féministe engagée dans l'organisation de la marche féministe du 8 mars déclare ainsi : "C'est une conférence de presse en fait. Une conférence de presse, tu peux la faire partout et tout le temps, sans demander d'autorisation. Il n'y a aucune différence entre la marche nocturne [féministe] et une conférence de presse. Simplement, il y a 40000 personnes qui s'y joignent $»^{23}$. Le choix de la localisation de ces «conférences», et de toute action militante dans l'espace public en général, est 
déterminé par la fonction symbolique des lieux. Fabrice Ripoll (2005a) distingue ainsi trois grandes significations, non exclusives. D'abord, choisir un géosymbole ${ }^{24}$ de lutte sert à 1) construire/activer l'identité du groupe mobilisé. Les marches féministes, auxquelles se joint le Forum des femmes de Yoğurtçu, ont ainsi lieu sur l'avenue Istiklal menant à la place Taksim, hauts-lieux de la gauche à Istanbul ${ }^{25}$. Ensuite, la localisation peut être choisie 2) en fonction de la cause soutenue. Le lieu symbolise alors cet enjeu (Ibid. : 589). Par exemple, la Protection des Forêts du Nord tient régulièrement des conférences dans les espaces naturels menacés par des projets urbains ou les quartiers touchés par la transformation urbaine. Enfin, la localisation sert à 3) symboliser la confrontation et à désigner un responsable, par exemple lorsque les conférences de presse ont lieu devant les bâtiments des autorités publiques, comme les municipalités d'arrondissement, afin de contester des politiques spécifiques. Par ailleurs, les conférences de presse sont également l'occasion de la production d'un marquage symbolique, visuel et sonore (pancartes, banderoles, slogans) en vue de réclamer un droit à prendre la parole (Ibid. : 592 et 599).

Les collectifs ont également recours à d'autres modes d'actions dans l'espace public, tels que la distribution de tracts pour encourager les passantees à participer à des actions qu'ils organisent, la collecte de signatures ou encore la vente d'objets estampillés du nom du collectif ou de slogans promouvant les causes qu'ils défendent, apportant ainsi une source de financement à leur action.

Par ailleurs, en fonction de leurs ressources, les collectifs peuvent s'adresser à différents échelons étatiques. Le collectif de la Protection des Forêts du Nord, qui est sans doute l'un des collectifs post-Gezi qui dispose de la plus grande capacité d'action ${ }^{26}$, organise ainsi des actions contre des projets urbains à l'échelle du quartier, dans les arrondissements centraux d'Istanbul mais aussi dans des arrondissements ultrapériphériques stambouliotes, et dans une moindre mesure dans d'autres régions de Turquie. Par ailleurs, le collectif intente des procès contre le gouvernorat d'Istanbul, tel ceux contestant le creusement de deux carrières de pierre (non loin des villages d'Ağaçlı et d'Işıklar, arrondissement d'Eyüp) servant à alimenter la construction du $3^{\mathrm{e}}$ aéroport d'Istanbul, en raison des risques environnementaux qu'elles suscitent. Il participe également à des rencontres entre divers groupes écologistes - autres collectifs stambouliotes et d'autres villes de Turquie, associations officielles turques d'envergure nationale - qui ont pour objectif de coordonner leurs actions afin d'améliorer la portée de leur action. En dernier lieu, si l'espace public physique est difficilement accessible, les collectifs mobilisent les réseaux sociaux (Facebook, Twitter, blogs) afin de prendre position sur des sujets d'actualité politique et diffuser ainsi leurs contre-discours. Malgré la situation autoritaire, les collectifs parviennent ainsi à investir divers espaces publics, choisis selon leur fonction symbolique, en développant des stratégies d'adaptation à ces contraintes, et en mobilisant des ressources leur permettant d'interpeller différents échelons de pouvoir dont ils connaissent les prérogatives.

\section{Les limites de la contestation publique en situation autoritaire}

L'évolution de la situation entre 2013 et 2018 a néanmoins participé à la baisse des activités militantes des collectifs dans l'espace public. C'est notamment dû à l'incertitude empêchant de véritablement mesurer les gains et risques potentiels de 
l'activité militante. Incertitude sur les risques juridiques d'abord, dans la mesure où l'état d'urgence instauré en 2016 est encadré par un court paragraphe de la constitution de 1982, et laisse ainsi libre à l'interprétation des accusations comme "l'appartenance, connexion ou contact avec une organisation terroriste", dans la mesure où il n'y a aucune définition juridique claire des termes employés (rapport de l'ICJ, $2018: 25)$. Incertitude aussi au regard de l'intégrité physique des militantee-s: la vague d'attentats de la période 2015-2017 a montré l'incapacité de l'État à prévenir de telles attaques voire laisse planer la suspicion d'une certaine passivité, décourageant les militants'e-s à se joindre à des événements contestataires ${ }^{27}$. L'incertitude concernant les gains semble toutefois avoir un moindre impact sur les militant·es, qui perçoivent souvent la présence dans « la rue » comme essentielle ${ }^{28}$. Par ailleurs, certaines victoires sont remportées par les collectifs à différents échelons, qu'il s'agisse d'entente tacite avec les maires de quartier (muhtar) lorsque cela concerne des micro-mobilisations, ou plus encore de réussites juridiques dans des démarches menées auprès de la municipalité métropolitaine d'Istanbul ou de ministères pour contester des projets urbains controversés. Néanmoins, les délais de telles démarches sont longs et les revers juridiques possibles. Par exemple, l'un des procès concernant les carrières de pierre a été perdu, et si l'autre a été remporté par le collectif écologiste, l'État a souvent recours à des stratégies juridiques pour outrepasser les décisions de justice.

17 En outre, les « conférences de presse » demeurent extrêmement cadrées et contrôlées, à la fois dans leur déroulement et dans leur contenu, et ne peuvent donc pas être considérées comme l'expression d'un usage autonome de l'espace (Ripoll, 2005b:10), particulièrement en situation autoritaire. Ainsi, l'organisation spatiale des acteurs en présence lors des «conférences de presse » traduit des rapports de domination, qui limitent souvent les possibilités d'élargissement du groupe contestataire. En effet, si ce mode d'action contourne les interdictions, il se déroule selon une ritualisation des positionnements des corps et des mouvements. Les militantees font toujours face à des policier-e's en civil filmant visages et déclarations, parfois à des forces de sécurité, autant d'intimidations qui entraînent une partition des sympathisante-s, certain'e-s préférant regarder de loin ou prendre des photographies pour les partager sur les réseaux sociaux plutôt que de se positionner derrière la banderole. Ces actions, strictement contrôlées et in fine ritualisées, posent la question de leur efficacité. Par ailleurs, comme Gilles Doronsorro (2005) le fait remarquer, l'État turc est peu sensible aux différentes formes de contestation, et les ouvertures démocratiques sont bien plus le fait des pressions extérieures, comme celles de l'Union Européenne. Enfin, il faut noter l'hétérogénéité des pratiques autoritaires selon les causes défendues, les acteurs impliqués, les modes d'action et les temporalités. Par exemple, la cause féministe et la cause écologiste demeurent relativement tolérées par le gouvernement, dans la mesure où il les mobilise en fonction de l'actualité politique, en les vidant toutefois de leur radicalité2 ${ }^{2}$. Dès lors, des manifestations féministes d'ampleur comme celles du 8 mars et du 25 novembre ont pu se tenir jusque récemment (répression du 25 novembre 2018 et du 8 mars 2019), même si elles portent des messages critiquant le traitement de la question du droit des femmes et des minorités sexuelles et de genre par l'État. De la même manière, les conférences de presse de la Protection des Forêts $d u$ Nord se tiennent globalement encore sans conflictualité. Tout autre est le traitement des contestations portant sur la question kurde, les guerres menées par l'État (guerre civile urbaine entre le PKK et l'État dans le Sud-Est à l'été 2015, guerre à Afrin, Kurdistan syrien, en 2018) ou encore les limogeages de fonctionnaires dans le cadre de l'état d'urgence (contestés par 
exemple par la grève de la faim menée par les universitaires et enseignants Nuriye Gülmen et Semih Özakça), quel que soit le mode d'action (manifestation de rue, expression sur les réseaux sociaux). L'autoritarisme ne s'applique donc pas de manière monolithique. Les militantees en ont bien conscience et adaptent en conséquence leur contre-discours, sauf à prendre le risque - parfois calculé et désiré pour médiatiser une cause - d'interpellations, de poursuites judiciaires voire d'incarcération.

En somme, si les militantees investissent toujours l'espace public malgré la situation autoritaire, les contre-discours y restent extrêmement contrôlés. Dans ces conditions, les collectifs déploient d'autres stratégies : ils ont créé des espaces semi-publics, dont ils ont un usage exclusif et autonome, pour poursuivre la production et la diffusion de leurs idées.

\section{Les modalités de production de contre-espaces publics}

\section{Inscription spatiale des contre-espaces publics dans les interstices de la ville autoritaire}

Dans la mesure où l'espace public est difficilement accessible, les acteurrrice's de l'opposition s'adaptent à la ville autoritaire afin de poursuivre la production, la diffusion et la mise en pratique de contre-discours. Ainsi, après la dispersion des manifestantees du mouvement du parc de Gezi, la tenue de forums et la création de collectifs qui en a découlée, ceux-ci ont cherché à créer des "espace[s] collectif[s] disponible[s] pour l'action » (Bulle, 2013), qui échappent à la production de l'espace liée au discours officiel dominant. Là, non seulement des formes d'expression contestataires plus abouties sont possibles, mais celles-ci peuvent également être concrètement mises en œuvre, créant ainsi des espaces contestataires dans la ville, qui à l'échelle microlocale, visent à mettre en pratique l'idéal de société auquel les militante's aspirent.

Les militante's ont ainsi créé ou investi des lieux spécifiques dans l'espace urbain. Ceux-ci sont utilisés par les collectifs de façon permanente lorsqu'il s'agit de squats, potagers urbains, coopératives alimentaires, cuisines communautaires, ou même une usine autogérée. Ils sont aussi pratiqués temporairement lorsqu'il s'agit de locaux d'associations officielles, de salles municipales louées pour l'occasion, de cafés sympathisants. $\mathrm{Au}$ sens juridique, ces espaces sont privés: par différentes combinaisons, les collectifs louent, partagent, s'entre-prêtent des locaux dans lesquels des réunions peuvent se tenir, des manifestations se préparer, des événements s'organiser. Ainsi, la Protection des Forêts du Nord s'est constituée en association officielle afin de pouvoir louer un local dans un quartier central d'Istanbul. Le Forum des femmes de Yoğurtçu tient des réunions dans les locaux d'une association proche d'un groupe politique d'extrême-gauche. Certains collectifs partagent le loyer d'un bâtiment officiellement loué par une association apolitique. D'autres tiennent leurs réunions dans des cafés sympathisants, louent des salles de spectacle ou de municipalités acquises au parti d'opposition CHP. Enfin, quelques collectifs ont occupé illégalement des bâtiments vides, créant des squats culturels qu'ils ont mis à disposition d'autres collectifs. Ces espaces sont néanmoins des espaces publics, au sens où ils sont ouverts et accessibles à tou'te's, ou en tout cas sont voués à l'être. Les collectifs formés sont des groupes ouverts à toute nouvelle participation, et partagent les informations 
concernant leurs réunions et leurs activités sur les réseaux sociaux, qu'il s'agisse de Twitter, Facebook, ou encore de groupes Gmail, invitant chacune à participer. Néanmoins, en tant qu'ils sont ouverts, ces espaces sont aussi soumis à une forme de contrôle: d'une part, microphones indésirables et personnes infiltrées sont des éventualités qui obligent à les fermer à des participant·e's soupçonnée's de proximité avec des groupes illégaux ou armés, d'autre part, si les participant·e's sont invitée's à participer aux débats politiques, une certaine base d'idéaux communs est attendue. Les militante's ont donc un monopole dans la définition des accès et des usages légitimes (Ripoll, 2005a: 362). Ce sont donc des espaces semi-publics, qui s'insèrent de façon ambivalente dans le paysage urbain. Certains collectifs veulent donner aux lieux une visibilité pour encourager la participation, et apposent ainsi à l'entrée des affiches relayant la tenue d'événements des milieux militants, des graffitis rappelant le mouvement du parc de Gezi, des enseignes avec des marqueurs politiques de gauche, tandis que d'autres souhaitent que ces lieux demeurent cachés : ce ne sont alors que de simples portes fermées qui ne disent rien des activités qui s'effectuent derrière elles.

La plupart de ces espaces sont concentrés dans les arrondissements centraux d'Istanbul ${ }^{30}$. Ce choix de localisation s'explique par diverses raisons. D'une part, certains arrondissements centraux, comme ceux de Kadıköy et de Sarıyer, sont traditionnellement acquis à la gauche dans son acception large. L'arrondissement de Beyoğlu, quant à lui, a été au cœur du mouvement du parc de Gezi ; c'est aussi là que sont situés les lieux de contestation historiquement symboliques comme la place Taksim et l'avenue Istiklal, et cet arrondissement a de ce fait connu une effervescence politique particulièrement intense après Gezi. Créer de tels espaces dans ces quartiers permet donc d'avoir "pignon sur rue» pour l'organisation de manifestations (Ripoll, 2005b : 11). C'est le cas par exemple du local utilisé par les collectifs féministes et LGBTQ+ stambouliotes, situé dans une rue perpendiculaire d'Istiklal. D'autre part, ces arrondissements sont plus facilement accessibles pour réunir les militant·ess et le public intéressé. Ceux-ci habitent en effet souvent dans le même arrondissement ou ceux avoisinants, dans la mesure où ils sont majoritairement issuees de la nouvelle classe moyenne et peuvent s'y loger sans difficulté financière. Cela dit, l'accès aux quartiers centraux est un défi pour les collectifs, car les loyers y sont particulièrement élevés. Ils parviennent néanmoins à contrecarrer « l'impossibilité d'accéder à l'espace pour toute autre forme d'activité qui ne génère pas de profit » (Bresnihan \& Byrne, 2015). Ce choix de localisation dans les quartiers centraux présente donc des difficultés, la première étant financière. Les deux collectifs étudiés ici parviennent par différentes stratégies à s'acquitter de leurs frais (loyer, charges, dépenses matérielles). La Protection des Forêts $d u$ Nord se finance grâce à des donations régulières et à l'organisation de concerts de soutiens ponctuels, quand le Forum des femmes de Yoğurtçu tire parti de ses bonnes relations avec l'association dont elle utilise le local et s'acquitte de dons réguliers pour la soutenir. D'autres collectifs font néanmoins face à l'augmentation de loyers dans des quartiers gentrifiés et sont parfois menacés de déménagement. Ces menaces présentent des enjeux de taille : elles entraînent la disparition d'espaces permettant l'organisation d'activités contestataires dans les centres urbains, et entament donc la restructuration politique de quartiers. Ainsi, l'arrondissement de Beyoğlu, dont l'effervescence politique post-Gezi a été mentionnée, se vide peu à peu de ses espaces alternatifs, face à une politique urbaine guidée par des idéaux de conservatisme et de consumérisme, dans la lignée du projet politico-social de l'AKP. D'autres dépensent beaucoup d'énergie 
à assurer la durabilité de leur local, mais cela prend le pas sur leurs activités contestataires.

22 La situation autoritaire n'épargne pas non plus ces espaces semi-publics. Des collectifs font parfois face à des intimidations de la part de l'État - descentes de police lors de l'organisation de certains événements - ou à la fermeture pure et simple des lieux utilisés, comme les locaux d'associations affiliées à l'extrême-gauche, ou encore d'un squat culturel qui a finalement subi une éviction (et dont les liens avec le mouvement de Gezi ont été dénoncés par les médias pro-gouvernementaux). De la même manière, les municipalités d'opposition enclines à prêter les salles municipales pour la tenue de réunions aux dimensions politiques sont devenues davantage fébriles après la promulgation de l'état d'urgence. Enfin, l'État peut aussi opérer une réappropriation des espaces créés. L'exemple des potagers urbains militants, créés afin de contester le système de production et de consommation capitaliste, est particulièrement évocateur : quelques années après, ceux-ci ont été institutionnalisés par les municipalités, dans la mesure où ils permettent de mettre en avant leur souci écologique et contribuent à la valorisation immobilière du quartier (Fautras, 2016). Les espaces utilisés pour les activités contestataires présentent donc tous un caractère éphémère, du fait des difficultés à les maintenir dans la ville autoritaire.

\section{Des espaces de production, de mise en pratique et de diffusion de contre-discours}

23 Dans sa thèse, Paul Cormier (2016) évoque l'utilisation de tels espaces par des militant·e's de gauche dans le contexte répressif des années 1980, montrant ainsi qu'ils ne sont pas nouveaux et se retrouvent dans des contextes similaires. Selon lui, «l'espace géographique, dans la mesure où il est soustrait temporairement ou durablement aux autorités, devient lieu de concentration des ressources organisationnelles» (Cormier, 2016: 134). Ces espaces sont ainsi à la fois des basesarrière organisationnelles et des espaces appropriés, qui permettent aux militante's de formuler et de mettre en pratique des discours suivant leur propre idéologie.

Ces espaces permettent aux collectifs d'organiser leurs actions militantes. Ainsi, d'une part, les militantees y préparent des manifestations en fonction de l'ordre du jour politique : recherche de nouveaux slogans, préparations de pancartes, distribution des tâches, intégration de nouveaux.elles militantees. Il s'agit aussi d'organiser la célébration de journées politiques comme l'anniversaire du mouvement du parc de Gezi ou encore le $1^{\text {er }}$ mai, des réunions hebdomadaires pour prévoir l'agenda des actions à venir, ou encore des concerts de solidarité pour des militantees emprisonnée'es. D'autre part, ils mènent des réflexions pour faire avancer les analyses des mécanismes de domination envers les groupes subordonnés, développent une expertise sur les leviers juridiques et décisionnels impliqués dans la fabrique de la ville, et questionnent l'interprétation de leurs propres identités, et ce à travers diverses activités : débats sur des sujets sociaux et politiques, rencontres avec des militantees venues de différentes villes de Turquie ou de l'étranger, projections de films engagés, portant sur des questions urbaines, environnementales, alimentaires ou sur l'identité sexuelle et de genre. appropriation «matérielle», soit une occupation avec usage exclusif (réservée à ces 
collectifs et aux participant·es) et un contrôle matériel de l'espace, qui permet d'en avoir un usage autonome, c'est-à-dire que les militante-es choisissent la manière de les utiliser et les régissent selon leurs propres normes (Ripoll, 2005b:8 et 11). Il n'y a cependant "pas d'appropriation sans marquage de l'espace » (Veschambre, $2004: 73$ ), marquage matériel mais aussi symbolique, comme dans le cas des conférences de presse dans l'espace public. Ainsi, ces espaces sont décorés par différents éléments qui rattachent les collectifs à leurs idéaux : les murs sont tapissés d'affiches véhiculant les valeurs défendues par les militante's (anti-frontiérisme, solidarité, antifascisme), les actions passées sont valorisées par des photographies de manifestation ou des affiches informatives sur des événements qu'ils ont organisés, des éléments font le lien avec le mouvement de Gezi. Autant de marqueurs qui constituent un socle commun de valeurs et inscrivent leur action dans des luttes qui participent à forger leur identité. Ce sont ainsi des marqueurs d' "affirmation» et de "légitimation» des collectifs (Ripoll et Veschambre, $2005: 6$ et 7).

Enfin, ces espaces sont également appropriés politiquement, par les règles qui les régissent. Celles-ci sont décidées collectivement et régulièrement questionnées et redéfinies lors de réunions, afin qu'elles correspondent aux valeurs défendues par les collectifs. Ainsi, les équipements disponibles tels que des lieux de réunion, cuisine, salle d'eau, parfois librairie, peuvent être utilisés par tou'te's, tout comme le matériel disponible (mégaphones et instruments de musique, projecteur, réseau internet, etc.). Les repas cuisinés sont vendus selon un prix libre, ou par un système de dons; des trocs de vêtements qui ont été préalablement donnés sont aussi organisés; les circuitscourts, l'économie locale et le soutien aux initiatives des collectifs (vente de produits textiles fabriqués par un collectif de femmes migrantes par exemple) sont valorisés. Enfin, les manières de se comporter au sein de ces espaces sont régis par des règles négociées à l'intérieur des collectifs, qui sont souvent affichées à l'entrée. Ainsi, les relations sociales se veulent inclusives : tout propos raciste, sexiste, homophobe est proscrit, la diversité des sympathies politiques est valorisée - bien qu'un socle commun d'opposition, ancré à gauche, soit bien tangible. Concernant les modes de communication, les mécanismes de discussion et de prise de décision ne doivent souffrir d'aucune forme de hiérarchie, que ce soit par genre, race, âge, expérience ou ancienneté. L'organisation spatiale choisie témoigne de cette ambition: les réunions s'effectuent toujours en cercle, la modération et la prise de note doivent être assurées par différentes personnes à chaque réunion, et une attention particulière est portée à la répartition de la parole entre hommes et femmes, ancien'ne's et nouveaux'elles venu'e's.

Il s'agit en somme d'une appropriation et d'une production idéologique de ces espaces, par opposition à l'espace public officiel produit par l'idéologie dominante : les règles sont pensées pour dépasser les relations économiques et sociales produites par la société capitaliste et conservatrice ; elles sont basées sur des valeurs de solidarité, de coopération, d'horizontalité, d'inclusion, de partage de connaissances et de compétences, de participation, de rencontre, de création. En somme, les militantees des collectifs appliquent de façon concrète les principes qui guident la société à laquelle ils aspirent. En ce sens, ces espaces se rapprochent de ce que Foucault appelait des "hétérotopies", car ils sont produits dans des lieux "qui sont des sortes de contreemplacements, sortes d'utopies effectives réalisées ", où " les hommes se trouvent dans une sorte de rupture absolue avec leur temps traditionnel » (Foucault, 1984:47 et 48). En effet, ils permettent d'expérimenter un temps situé hors de l'ordre dominant qui régit les interactions sociales, politiques et économiques en Turquie. Ce sont 
néanmoins bien des expérimentations fragiles, qui soulèvent des questionnements et nécessitent sans cesse des réajustements, car elles sont traversées de part en part par des logiques capitalistes. Comment un collectif peut-il financer ses activités sans dégager de profit? Quelle place accorder à l'esthétique dans la diffusion de leurs activités, sans reproduire des stratégies marketing? La mise en œuvre d'interactions sociales inclusives ne va également pas toujours de soi et des rappels - via des ateliers ou des discussions autour d'un litige - sont souvent effectués pour lutter contre la reproduction de rapports de domination. Autant de contradictions qui émergent dans l'organisation de ces espaces et qui sont parfois difficiles à gérer par les collectifs.

La diffusion des contre-discours produits passe aussi par la mise en réseau de ces collectifs, notamment via l'utilisation de ces contre-espaces publics. Une des tâches que se fixent les collectifs est justement de constituer et de maintenir un réseau de groupes d'opposition qui implique différentes échelles et différents types d'acteurs. Ainsi, les collectifs planifient des rencontres entre eux et organisent des actions contestataires communes dans l'espace public officiel et dans les contre-espaces publics, à l'échelle métropolitaine essentiellement, mais aussi à l'échelle nationale voire internationale, lorsqu'ils organisent par exemple des rencontres entre collectifs et associations officielles écologistes de différentes provinces de Turquie, ou en invitant des militante's de France, de Grèce ou encore d'Afrique du Sud pour échanger sur leurs expériences. Par ailleurs, les collectifs développent aussi d'étroits liens avec différents acteurs de la ville, qu'il s'agisse de relations informelles avec les maires de quartier pour bénéficier de certaines ressources, de sollicitations envers les maires d'arrondissement qui aboutissent notamment du fait de leur appartenance à un grand parti d'opposition, ou encore de collaboration académique avec des chambres professionnelles (urbanistes et architectes en premier lieu) qui apportent une expertise primordiale sur les luttes urbaines. Enfin, les collectifs disposent aussi de moyens de diffusion de leurs discours et pratiques contestataires sur différents canaux : contacts dans les journaux d'opposition en format papier ou numérique qui relaient leurs déclarations et actions, rédaction de magazines et fanzines de l'extrême-gauche et de la mouvance anarchiste, programmes sur des radios indépendantes invitant des militante's et des universitaires à commenter l'actualité politique, organisation de journées d'étude sur les luttes urbaines, etc. Autant de moyens de diffusion et de mises en réseau des collectifs et des contre-espaces publics qu'ils utilisent.

\section{Conclusion}

Cette recherche tend donc à apporter quelques éclairages sur l'action collective en situation autoritaire et sur sa dimension spatiale. D'abord, l'étude de la période suivant la répression d'un mouvement social permet de montrer qu'il existe des formes de continuation. Les collectifs étudiés revendiquent ainsi leur filiation à la fois idéelle et organisationnelle avec le mouvement du parc de Gezi. On peut même la qualifier d'affective, tant ce moment constitue une étape clé de la politisation des militantees et se voit sans cesse remémoré avec nostalgie dans les discours. Ces collectifs ne constituent toutefois qu'une forme de continuation de Gezi : ils ne représentent qu'un fragment de la grande diversité de causes, de personnes mobilisées et d'origines géographiques et sociologiques, qui ont constitué le mouvement du parc de Gezi. Par ailleurs, nous avons vu que si la situation autoritaire systémique limite largement 
l'activité militante dans l'espace public, l'étude plus fine des pratiques autoritaires montre qu'elle ne s'applique pas de façon homogène, et que certaines actions peuvent toujours être menées, dans le cadre d'une "conflictualité négociée avec l'État" (Geoffray, $2011: 34$ ).

Dans un tel contexte, l'activité militante tend toutefois à être davantage menée dans des contre-espaces publics, dont les modalités de création permettent de rester relativement en dehors du contrôle de l'État. Ces espaces permettent ainsi aux militante-s d'organiser les actions menées dans l'espace public, de discuter et ainsi faire progresser leurs réflexions politiques, de partager leurs idéaux avec les participant·es dans une logique de politisation à l'échelle locale, et d'expérimenter le modèle de société auquel ils aspirent. Entre 2013 et 2018, ces espaces ont eu tendance à diminuer, d'une part du fait des difficultés à les maintenir dans les centre-villes gentrifiés, d'autre part parce que les collectifs se défont parfois, du fait de conflits internes, d'arrestations ou de migrations de militante-s hors de Turquie. Enfin, l'efficacité politique de ces collectifs reste limitée : leurs actions pour contrecarrer la fabrique de la ville actuelle ne mènent que rarement à des victoires juridiques et politiques tangibles, et les contreespaces publics créés, dans les interstices de la ville, ne semblent pas fortement inquiéter l'État, qui tolère globalement leurs activités. Toutefois, l'existence et le maintien de tels collectifs permet de faire le lien entre deux cycles de mobilisation, dans la mesure où ils permettent la «[mise] à disposition des réseaux activistes, un répertoire de buts et de tactiques et une identité collective" (Taylor, $1989: 245$ ). C'est en effet le bouche-à-oreilles rapide entre militantees (Danış, 2013) qui a permis la première journée de mobilisation dans le parc de Gezi en 2013, témoignant de l'existence d'un réseau " en veille ». De même, en élaborant et expérimentant une vie sociale alternative au sein des contre-espaces publics, les collectifs esquissent peut-être ainsi la possibilité d'une transformation sociale à long terme.

\section{BIBLIOGRAPHY}

AYMÉ P. (2017), Engagement féminin conservateur et inclusion à la sphère politique. Le cas de la branche féminine du Parti de la Justice et du Développement en Turquie, Mémoire de Science politique, sous la direction d'Élise Massicard, Institut d'Études politiques de Paris, 119 p.

BILGIÇ E. E., KAFKASLI Z. (2013), Gencim, özgürlükçüyüm, ne istiyorum? \#direngeziparkı [Je suis jeune, je suis pour la liberté, qu'est-ce que je veux? \#leparcdeGezirésiste], Anket Sonuç Raporu [Rapport des résultats d'enquête], Bilgi Üniversitesi Yayınları, Istanbul, 30 p. http://www.bilgiyay.com/ Content/files/DIRENGEZI.pdf (consulté le 20.02.2018)

BONNEMAISON J. (1981), « Voyage autour du territoire », L'Espace géographique, Tome 10, n4, pp. 249-262.

BOZARSLAN H. (2004), Histoire de la Turquie contemporaine, Paris, La Découverte, collection « Repères », 123 p. 
BRESNIHAN P., BYRNE, M. (2015), "Escape into the City: Everyday practices of Commoning and the Production of Urban Space in Dublin", Antipode, vol. 47, n¹, pp. 34-54.

BROOKER P. (2009), Non-Democratic Regimes. Theory, Government and Politics, Red Globe Press, “ Comparative Government and Politics", $304 \mathrm{p}$.

BULLE S. (2013), « Pourquoi la rue inspire-t-elle la révolte ? Compétences émeutières et projet de transformation sociale dans le mouvement d'occupation mondial (Espagne, Israël, USA) » [traduit de l'anglais par Sharon Winkler Moren], Justice spatiale / spatial justice, $n^{\circ} 5$. http:// www.jssj.org/wp-content/uploads/2013/09/JSSJ5-8fr1.pdf (consulté le 04.04.2018)

ÇALIŞKAN K. (2018), “Toward a new political regimein Turkey: From competitive toward full authoritarianism”, New Perspectives on Turkey, n¹8, pp. 5-33.

CASTELLS M. (1983), The City and the Grassroots: A Cross-Cultural Theory of Urban Social Movements, University of California Press, Berkeley, $471 \mathrm{p}$.

CORMIER P. (2014), « La gauche en Turquie : une histoire fragmentée », Ifri, note franco- turque $\mathrm{n}^{\circ} 11,22 \mathrm{p}$

CORMIER P. (2016), Les conséquences biographiques de l'engagement en contexte répressif: militer au sein de la gauche radicale en Turquie : 1974-2014, Thèse en Science politique, Institut d'Études Politiques de Bordeaux, Université de Lausanne, $535 \mathrm{p}$.

DANIş T. (2013), Yasiam için, Gezi Bostan direnisio ve mes.

DIKEÇ M. (2009), « L'espace, le politique et l'injustice », Justice spatiale / spatial justice, $\mathrm{n}^{\circ} 1$. https:// www.jssj.org/article/lespace-le-politique-et-linjustice (consulté le 15.03.2018)

DORONSORRO G. (2005), La Turquie conteste: Mobilisations sociales et régime sécuritaire, CNRS Éditions, « Moyen-Orient », Paris, 247 p.

DORONSORRO G. (2017), « L'État-AKP et le gouvernement par la crise », Mouvements, vol. 2, n90, pp. 11-18.

DOUAY N. (2012), « L'activisme urbain à Montréal : des luttes urbaines à la revendication d'une ville artistique, durable et collaborative », L'Information géographique, vol. 76, n³, pp. 83-96.

ERDI LELANDAIS G. (2005), Altermondialistes en Turquie. Entre cosmopolitisme politique et ancrage militant, Paris, L'Harmattan, «États, pouvoirs et sociétés », 377 p.

ERDI LELANDAIS G. (2017), « L'urbanisation néolibérale et conservatrice au prisme de l'autoritarisme en Turquie ", Mouvements, vol. 2, n90, pp. 54-61.

ERIKSON R. S., TEDIN K. L. (2003), American Public Opinion: Its Origins, Content and Impact, Routledge, New York, 400 p. - cité dans LOST J. T., FEDERICO C. M., NAPIER J. L. (2009), "Political Ideology: Its Structure, Functions, and Elective Affinities”, Annual Review of Psychology, n60, pp. 307-337.

ESEN B., GÜMÜşÇÜ ş. (2016), "Rising competitive authoritarianism in Turkey", Third World Quarterly, vol. 37, n9, pp. 1582-1584.

FAUTRAS A. (2016), « Les nouveaux bostan d'Istanbul : quelle pérennisation pour les jardins de la contestation?», European Journal of Turkish Studies, n²3. http://journals.openedition.org/ejts/ 5400

FOUCAULT M. (1984), « Des espaces autres. Hétérotopies », Conférence au Cercle d'études architecturales, dans Architecture, Mouvement, Continuité, n5, pp. 46-49. 
FRASER N. [2001] (1992), « Repenser la sphère publique : une contribution à la critique de la démocratie telle qu'elle existe réellement ", in CRAIG. (ed.) (1992) Habermas and the Public Sphere, MIT Press, Cambridge, pp. 109-142.

GEOFFRAY M.-L. (2011), « Étudier la contestation en contexte autoritaire : le cas cubain », Politix, $\mathrm{n}^{\circ}$ 93, pp. 29-45.

GLASIUS M. (2018), "What authoritarianism is ... and is not: a practice perspective", International Affairs, $\mathrm{n}^{\circ} 3$, pp. 515-533.

GOURISSE B. (2014), « Une clé de lecture du politique en Turquie : les rapports État-Partis », Politix, n¹07, pp. 195-218.

HARVEY D. (2012), Rebel cities. From the Right to the City to the Urban Revolution, Verso, Londres, $206 \mathrm{p}$.

International Commission of Jurists (ICJ) (2018), Justice Suspended: Access to Justice and the State of Emergency in Turkey [rapport], Genève, $47 \mathrm{p}$.

KAYA S. (2017), « Anatomie de l'armée en Turquie après la tentative du coup d'État du 15 juillet $2016 »$, Mouvements, vol. 2, n90, pp. 19-29.

KEYDER Ç. (2013a), "Yeni Orta Sınıf" [La nouvelle classe moyenne], Bilimsel Akademisi. https:// bilimakademisi.org/wp-content/uploads/2013/09/Yeni-Orta-Sinif.pdf

KEYDERÇ. (2013b), “Law of the Father”, LRB Blog, Bilim Akademisi. http://www.lrb.co.uk/blog/ 2013/06/19/caglar-keyder/law-of-the-father/

LEFEBVRE H. [1974] (2000), La Production de l'espace, Anthropos, Paris, 512 p.

LEVITSKY S., WAY L. (2002), “The Rise of Competitive Authoritarianism”, Journal of Democracy, vol. $13, \mathrm{n}^{\circ} 2$, pp. $51-65$.

LEWIS D. (2013), “Civil Society and the Authoritarian State: Cooperation, Contestation and Discourse", Journal of Civil Society, vol. 9, n 3, pp. 325-340.

MASSICARD É. (2013), « Mais qui représente le mouvement de Gezi ? », Observatoire de la Vie Politique Turque, IFEA. http://ovipot.hypotheses.org/9903 http://ovipot.hypotheses.org/8910

MILLER B., NICHOLLS W. (2013), "Social Movements in Urban Society: the City as a Space of Politicization”, Urban Geography, vol. 34, n4, pp. 452-473.

MILLS A. (2006), "Boundaries of the Nation in the Space of the Urban: Landscape and Social Memory in Istanbul", Cultural Geographies, vol. 13, n³, pp. 367-394.

MONTABONE B. (2013), « Droit à la ville et contestation de l'ordre moral urbain en Turquie », EchoGéo, Sur le Vif. http://echogeo.revues.org/13567 (consulté le 15.03.2018)

MORVAN Y. (2013), «L'aménagement du grand Istanbul : entre ambition géopolitique mondiale et enjeux fonciers locaux. Le troisième pont sur le Bosphore", Hérodote, n¹48, pp. 197- 210.

Non-Attribué (NA) (2016), «Règles du jeu en « situations autoritaires », Journée d'étude, Calenda [en ligne] https://calenda.org/359862 (consulté le 02.11.2018)

NICHOLLS W. J. (2008), “The urban question revisited: the importance of cities for social movements", International Journal of Urban and Regional Research, vol. 32, n4, pp. 841-859. PÉROUSE J.-F. (2013a), « Le parc de Gezi : dessous d'une transformation très politique », Métropolitiques. https://www.metropolitiques.eu/Le-parc-Gezi-dessous-d-une.html 
PÉROUSE J.-F. (2013b), « Hybristanbul. Les grands projets d'aménagement urbain en Turquie », La Vie des idées. https://laviedesidees.fr/Hybristanbul.html

PÉROUSE J.-F. (2014), « Réinterroger le quartier, à partir d'Istanbul en proie aux transformations », IFEA. http ://www.ifea- istanbul.net/index.php ?

option=com_k2\&view=item\&id=3190 :réinterroger-le-quartier-à-partir-distanbul-en-proie-auxtransformations\&Itemid=537 (consulté le 05.06.2018).

PÉROUSE J.-F. (2017), « Les événements de Gezi, ou le début de la dérive autoritaire, vu des périphéries », Mouvements, vol. 2, n90, pp. 54-61.

PIRON S. (2013), "Contexte, situation, conjoncture », Des contextes en histoire, Centre de recherches historiques, Actes du Forum du CRH (2011), pp. 27-65.

PLANEL S. (2015), « Espaces autoritaires, espaces (in)justes ? ", Justice spatiale / spatial justice, $n^{\circ} 8$. https://www.jssj.org/article/espaces-autoritaires-espaces-injustes/

PLEYERS G., GLASIUS, M. (2013), « La résonance des « mouvements des places » : connexions, émotions, valeurs ", Socio, n², pp. 59-80.

RIPOLL F. (2005a), La dimension spatiale des mouvements sociaux. Essais sur la géographie et l'action collective dans la France contemporaine à partir des mouvements de "chômeurs » et " altermondialistes ", Thèse de Géographie, Université de Caen Basse-Normandie, 741 p.

RIPOLL F. (2005b), «S'approprier l'espace... ou contester son appropriation ? Une vue des mouvements sociaux contemporains ", Norois, $n^{\circ} 195$, pp. 29-42.

RIPOLL F., VESCHAMBRE V. (coord.) (2004), « Appropriation », ESO. Travaux et documents de l'UMR 6590 , Séminaires « Appropriation » et « Habitat et stratégies résidentielles », n²1, mars, pp. 5-107.

SPURK J. (2016), « Mouvements de masse, espaces publics et contre-espaces publics », Les Cahiers d'EMAM, n²8. http://journals.openedition.org/emam/1272 (consulté le 11.05.2018)

ŞIMŞEK S. (2004), “New Social Movements in Turkey Since 1980”, Turkish Studies, vol. 5, n², pp. 111-139.

TAŞAN-KOK T., BAETEN G. (2012), Contradictions of Neoliberal Planning: Cities, Policies, and Politics, Springer, New York, $242 \mathrm{p}$.

TAYLOR V. (2005), « La continuité des mouvements sociaux. La mise en veille du mouvement des femmes », in FILLIEULE O. (dir.), Le désengagement militant, Belin, Paris, pp. 229-250.

ULDAM J., McCURDY P. (2013), 'Studying Social Movements: Challenges and Opportunities for Participant Observation”, Sociology Compass, vol. 7, n¹1, pp. 941-951.

UYSAL A. (2005), Le répertoire d'action de la politique dans la rue : les actions protestataires et leur gestion étatique en Turquie dans les années 1990, Thèse de doctorat en Sciences Politiques, Université Paris 1 Panthéon Sorbonne.

YEĞEN O. (2017), “Constitutional Changes under the AKP Government of Turkey”, Tijdschrift voor Constitutioneel Recht, $\mathrm{n}^{\circ} 1$, pp. 70-84.

\section{NOTES}

1. Soit les luttes qui ont « la ville comme objet et comme cadre » (Douay, 2012): pour des espaces de verdure et la création de zones de rassemblement en cas de tremblement de terre face à une 
urbanisation rapide des espaces vacants dans le tissu urbain, contre la transformation urbaine de quartiers conduisant à l'éviction des habitant·e·s, pour la protection du patrimoine architectural et culturel de la ville, etc.

2. "Başbakanın otoriter tavrının etkili"; "Polisin protestoculara uyguladığ orantısı gücün etkili"; "Demokratik hakların ihlal edilmesinin etkili" (toutes les traductions sont de l'auteure, A.F.).

3. Sümbül Kaya (2017) revient sur les raisons de cette ouverture démocratique : celle-ci s'inscrit dans le processus d'adhésion à l'Union Européenne, et a permis au gouvernement AKP de limiter le pouvoir de l'armée - qui a opéré plusieurs coups d'État ces dernières décennies - en restructurant ses institutions selon les critères européens. Le rapprochement avec l'UE a aussi permis d'obtenir des ressources financières extérieures (IDE, fonds européens) tout en choyant une opinion publique favorable à l'adhésion de la Turquie à l'UE (Yeğen, 2017).

4. Il faut toutefois bien souligner que l'État a recours à des stratégies juridiques qui permettent de contourner des résultats électoraux qui lui sont défavorables : publication d'un décret-loi en 2016 dans le cadre de l'état d'urgence permettant d'annuler la nomination de maires accusée-s de soutien au terrorisme (appliqué dans les régions kurdes contre le parti d'opposition du HDP) et de les remplacer par des administrateurs AKP (kayyum); en mai 2019, le Haut Conseil Électoral (YSK), prétextant des manquements dans la composition des bureaux de vote, décide de réorganiser l'élection de la Grande Municipalité d'Istanbul (IBB), remportée de justesse par le candidat d'opposition CHP. La réorganisation de l'élection en juin a conduit à une large victoire du candidat $\mathrm{CHP}$.

5. Gilles Doronsorro (2017) et Benjamin Gourisse (2014) ont montré les collusions entre État, parti, syndicats, médias et groupes économiques dans le système politique turc, créant un «Étatparti ». C'est notamment, mais pas uniquement, le cas sous l'AKP : déploiement du personnel politique et des militant.e's du parti dominant dans les institutions publiques de sorte qu'elles deviennent partisanes; modification des lois pour négocier des soutiens politiques ; mécanismes législatifs et administratifs pour favoriser les affaires de groupes économiques proches du pouvoir ; utilisation des positions institutionnelles pour le rachat de médias. Tout cela contribue à la faible autonomie des institutions.

6. On peut évoquer la levée d'immunité parlementaire des député'e·s visé'e·s par des procédures judiciaires en mai 2016, ciblant directement le HDP, parti d'opposition, aboutissant à l'emprisonnement d'une grande partie d'entre eux, notamment des deux co-président·ess du parti, Selahattin Demirtaş et Figen Yüksekdağ. On peut aussi citer les menaces de poursuites judiciaires régulièrement proférées par l'AKP à l'encontre du leader d'opposition kémaliste Kemal Kiliçdaroğlu, ou encore la récente organisation d'élections présidentielles et législatives anticipées, permettant entre autres de compliquer la participation aux élections de la candidate Meral Akşener, à la tête du İyi Parti (« le Bon Parti »), un nouveau parti d'opposition nationaliste. 7. “(...) to influence and shape the vast assemblage of oral and written texts, rituals, visual culture, and performance that constitute public discourse".

8. A "set of beliefs about the proper order of society and how it can be achieved".

9. Si depuis son arrivée au pouvoir, l'AKP a toujours eu un discours conservateur et islamique, c'est surtout à partir du début de l'été 2015 que celui-ci est devenu nationaliste, après l'entrée du HDP à la grande assemblée nationale lors des élections législatives du 9 juin. Notons aussi que si l'AKP a effectivement renforcé les réformes néolibérales entamées dans les années 1980 par Turgut Özal, il demeure très interventionniste dans des secteurs clés tels que l'économie et l'immobilier (Taşan-Kok et Baeten, 2012).

10. La place Taksim accueille en effet le Monument de la République (édifié en 1928), célébrant la fondation de la république turque en 1923 par Mustafa Kemal Atatürk. Quant au parc de Gezi, dessiné par l'urbaniste français Henri Prost sur invitation d'Atatürk, il est venu supplanter en 1940 les anciens baraquements d'artillerie datant 
de l'Empire ottoman. Il s'agissait donc de détruire le précédent récit urbain - un symbole de l'Empire ottoman - pour y ajouter un sens républicain, soit un monument symbolique et un projet de planification urbaine guidé par des principes dirigistes et modernistes, chers à Atatürk.

11. La période de 2013 à aujourd'hui est en effet jalonnée de divers autres événements (conflit ouvert entre l'État et la confrérie Gülen fin 2013, reprise du conflit entre l'État et le PKK à l'été 2015, criminalisation et emprisonnement des membres du HDP à l'automne 2015, vague d'attentats en 2015 et 2016, tentative de coup d'État à l'été 2016 et instauration de l'état d'urgence), qui entrainent une répression juridique de grande ampleur. Cela a pour effet d'augmenter fortement les risques de l'engagement.

12. Ces questions sont loin de représenter toutes les causes qui ont été portées dans le débat public lors du mouvement du parc de Gezi. Particulièrement, on notera l'absence de collectifs défendant spécifiquement les droits de minorités ethniques et religieuses, telles que les mouvements kurdes et alévistes. Cela peut s'expliquer par plusieurs raisons : ces causes sont déjà très structurées par des organisations dont il est difficile de s'autonomiser ; ces organisations ont par ailleurs un fonctionnement très hiérarchique qui sied mal à l'idéal antihiérarchique. Ces causes sont néanmoins présentes dans les discussions et les événements organisés par les collectifs, en fonction de l'actualité politique.

13. Nancy Fraser évoque des publics «subalternes", soit des groupes subordonnés («femmes, ouvriers, personnes de couleurs et homosexuelle's" ", écrit-elle) mais ce terme ne semble pas approprié dans le cas d'étude: si les causes défendues par les acteurrice's de l'opposition à Istanbul sont inaudibles, ils ne sont pas nécessairement des groupes subalternes stigmatisés. Voir plus loin : Qui sont les militante's des collectifs?.

14. Ce sont des projets titanesques, entrepris sous le gouvernement AKP, destinés à rendre la métropole attractive. On compte par exemple le troisième aéroport, en cours de construction, qui devrait être le plus grand du monde en terme de passagers (200 millions), le troisième pont sur le Bosphore, achevé en 2017, possédant la plus grande portée du monde entre deux pylônes, et qui participe à l'étalement urbain, ou encore l'hypothétique canal d'Istanbul, censé relier la mer Noire à la mer de Marmara pour désengorger le trafic maritime sur le Bosphore.

15. Toutefois, c'est aussi une échelle d'action primordiale pour l'AKP : la mobilisation électorale se fait grâce à des « comités de quartier » qui entretiennent des réseaux partisans et sont à la base des succès électoraux du parti (Aymé, 2017).

16. Formulation utilisée par une militante féministe ayant participé au mouvement du parc de Gezi (entretien du 21.06.2016).

17. Lors du coup d'État du 12 septembre 1980 orchestré par l'armée, institutions, partis politiques, syndicats et associations sont dissous. Pendant trois ans, le gouvernement militaire pourchasse, emprisonne, torture et exécute les militante-s des mouvements contestataires, principalement ceux de gauche. Ainsi, on a décompté plus de 650000 arrestations, dont 230000 déférées devant des cours martiales, et 50 exécutions officielles (Bozarslan, 2004).

18. Keyder explique ainsi que la Turquie compte plus de 200 universités avec 4 millions d'étudiant·e·s, et depuis 2008, 2,5 millions de diplômée'-s ont été ajoutée's à la population.

19. “(...) whose members work in relatively modern workplaces, with leisure time and consumption habits much like their global counterparts"

20. Cela s'inscrit dans un contexte global de migrations vers Istanbul et les grandes villes du pays à partir des années 1950, depuis différentes régions de Turquie et des Balkans.

21. Paul Cormier (2014) délimite ainsi la gauche turque, dans une acceptation large : la gauche sociale-démocrate du CHP, parti kémaliste, la gauche socialiste légale, et l'extrême-gauche révolutionnaire et clandestine. 
22. Les travaux d'Ayşen Uysal (2005), qui a enquêté sur les manifestations de rue en Turquie dans les années 1990, montrent bien les continuités dans cet autoritarisme étatique qui limite l'action des militant·e's dans l'espace public.

23. "Aslında bir basın açıklaması. Basın açıklaması, her zaman ve her yerde yapabilirsin, izin almadan. [Feminist] gece yürüyüşü basın açıklamasından hiçbir farkı yok. Sadece 40000 kişi katıldı" (entretien du 23 mars 2018).

24. «(..) un lieu, un itinéraire, une étendue qui, pour des raisons religieuses, politiques ou culturelles prend aux yeux de certains peuples et groupes ethniques, une dimension symbolique qui les conforte dans leur identité » (Bonnemaison, 1981 : 256).

25. L'avenue Istiklal a été le théâtre de nombreuses manifestations dont le parcours se terminait à l'emblématique place Taksim. Chère aux Kémalistes par la présence du monument de la République, elle est aussi importante pour la gauche turque, surtout depuis 1977 : alors que des partis d'extrême-gauche (TIP, TSIP, TKP) s'y étaient réunis pour célébrer le $1^{\mathrm{er}}$ mai en masse, des coups de feu furent tirés, créant une immense panique. Le bilan s'éleva à 37 morts. À l'issue de ce drame, les manifestations du $1^{\mathrm{er}}$ mai, d'une forte valeur symbolique pour la gauche, ont été interdites sur la place Taksim jusqu'à aujourd'hui, exception faite de 2009 à 2012.

26. C'est un collectif qui mène également des actions à l'échelle métropolitaine et nationale; il est composé d'une cinquantaine de membres (la fourchette haute des collectifs étudiés); il s'est fait connaître par son opposition aux mégaprojets et dispose ainsi d'une certaine renommée.

27. Par exemple, l'attentat de Suruç du 20 juillet 2015, ayant coûté la vie à 32 personnes, visait un événement de soutien à la ville de Kobanê, dans le Kurdistan syrien, alors assiégée par l'État islamique. L'événement avait réuni des militant·es de la gauche turque et du mouvement kurde.

28. Il faut noter que cette volonté de mener des actions « dans la rue » est associée à la gauche turque, parfois perçue d'ailleurs comme une «fétichisation» (entretien avec un militant des luttes urbaines, 12 mars 2018).

29. Par exemple, le meurtre de l'étudiante Özgecan Aslan en 2015, qui a ému toute la Turquie, a largement été condamné par les responsables politiques. Si les registres de l'État et du collectif du Forum des femmes de Yoğurtçu sont largement distincts, ils s'inscrivent tous deux dans la question des droits des femmes. Autre exemple, si la Protection des Forêts du Nord s'est opposée à la construction $\mathrm{du}$ troisième aéroport et $\mathrm{du}$ troisième pont sur le Bosphore, dénonçant le « massacre d'arbres » engendré et l'appel d'air à l'urbanisation massive des réserves forestières, le gouvernement a toutefois répondu que les arbres déracinés seraient tous replantés ailleurs, s'inscrivant ainsi lui aussi dans un discours écologiste.

30. Il va sans dire que ces quartiers n'ont pas l'apanage des activités politiques. Néanmoins, la majeure partie des collectifs étudiés formés après le mouvement du parc de Gezi s'organisent dans les espaces centraux stambouliotes.

\section{ABSTRACTS}

Starting from the example of the "Gezi Park" protest movement in Turkey (2013), this article aims to study the forms of continuation of a social movement after its suppression, and their upholding in a hostile political environment. Indeed, in Istanbul, after being expelled from the public spaces they occupied, protesters scattered into neighbourhoods and then created antihierarchical "collectives", acting on urban and social issues, thus extending certain demands and 
organizational forms developed during the movement. By a geographical approach, the article aims to analyse the spatial dimension of the activists' action strategies in an authoritarian situation. Activists observe a public space shaped by discourses and legislations stemming from a dominant socio-political project that carries an ideological dimension. They then seek on the one hand to produce counter-discourses in the public space to claim a right to speak and to try to influence the political discourse, even if their scope of action remains limited because of the authoritarian situation. On the other hand, they create counter-public spaces, in which they can avoid suppression, but also gather, organize, deepen their political reflections and propose alternative social, economic and political relations, while maintaining a network that can be mobilized in a more conducive political environment.

En partant de l'exemple du mouvement de contestation du « parc de Gezi » en Turquie (2013), cet article vise à étudier les formes de continuation d'un mouvement social après sa répression, et leur maintien dans un environnement politique hostile. En effet, à Istanbul, après avoir été expulsée's des espaces publics qu'ils occupaient, les manifestant·e's se sont dispersé'e-s dans les quartiers et ont ensuite créé des "collectifs" antihiérarchiques, militant sur des questions urbaines et sociales, prolongeant ainsi certaines revendications et modes organisationnels développés lors du mouvement. Par une entrée géographique, il s'agit ici d'analyser la dimension spatiale des stratégies d'action des militant·es dans une situation autoritaire. Les militant·es observent un espace public façonné par des discours et des législations issus d'un projet sociopolitique dominant à dimension idéologique. Ils cherchent alors d'une part à produire des contre-discours dans l'espace public pour revendiquer un droit à la parole et tenter d'influencer le discours politique, même si leur périmètre d'action demeure limité du fait de la situation autoritaire. D'autre part, ils créent des contre-espaces publics, dans lesquels ils peuvent éviter la répression, mais aussi se retrouver, s'organiser, approfondir leurs réflexions politiques et proposer des relations sociales, économiques et politiques alternatives, tout en maintenant un réseau qui pourra être mobilisé dans un environnement politique plus propice.

\section{INDEX}

Keywords: social movement, public space, counter-public spaces, authoritarian situation, Gezi Park movement, Turkey, Istanbul

Mots-clés: mouvement social, espace public, contre-espaces publics, situation autoritaire, mouvement du parc de Gezi, Turquie, Istanbul

\section{AUTHOR}

\section{AGATHE FAUTRAS}

Doctorante au Laboratoire Espaces, Nature et Culture (ENeC - Sorbonne Université)

Chercheuse associée à l'Institut Français des Études Anatoliennes (IFEA - Istanbul) 